\title{
Density conditions in Fréchet and (DF)-spaces
}

\author{
KLAUS D. BIERSTEDT and JOSE BONET
}

\begin{abstract}
We survey our main results on the density condition for Fréchet spaces and on the dual density conditions for (DF)-spaces (cf. [2] and [3], I.) as well as some recent developments.

At the end of section 1., we include a new result on the projective tensor product of two Fréchet spaces. Taskinen's construction of counterexamples to Grothendieck's "probleme des topologies" yields Fiechet spaces $E, F$ with the density condition such that $E \hat{B}_{x} F$ is not (even) distinguished (see [14]). We prove now that the negative solution of the "probleme des topologies" is, in fact, the only obstruction: For two Fréchet spaces $E$ and $F$ with the density condition, $E \hat{\otimes}_{\mathrm{x}} F$ has the density condition as well (and hence is distinguished) whenever the "probleme des topologies" has a positive solution for the pair $(E, F)$.
\end{abstract}

Distinguished locally convex (l.c.) spaces, that is, locally convex spaces $E$ such that the strong dual $E_{b}^{\prime}$ is barrelled, were introduced by J. Dieudonné and L. Schwartz. Later on, A. Grothendieck showed that a metrizable l.c. space $E$ is distinguished if and only if $E_{b}^{\prime}$ is bornological.

Let $\left(U_{n}\right)_{n \in N}$ denote a basis of 0 -neighborhoods for a metrizable l.c. space $E$ and define the "inductive dual" $E_{i}^{\prime}$ of $E$ by $E_{i}^{\prime}:=$ ind $_{n \rightarrow \infty} E_{i_{n}^{*}}^{\prime}$ Then $E_{i}^{\prime}$ carries a stronger topology than $E_{b}{ }^{\prime}$ and, in fact, $E^{\prime}$, is the bornological space associated with $E_{b}^{\prime}$. Hence $E$ is distinguished if and only if $E_{b}^{\prime}=E_{c}^{\prime}$.

Thus, the class of the distinguished Fréchet spaces is related to the naive idea that the strong dual of a Fréchet space $E$; i.e., the strong dual of a space which can be represented as a countable (reduced) projective limit of Banach spaces, should also be representable as a countable inductive limit of Banach spaces (and that the duality should work by simply dualizing the projective spectrum). As the existence of non-distinguished Fréchet spaces demonstrates (the first example of such a space was due to Köthe and Grothendieck and was, in fact, a certain Köthe echelon space $\lambda_{1}$ ), the naive idea does not work

1980 Mathematics Subject Classification (1985 revision): 46A06, 46A07, 46A09, 46A20, 46A32, 46A45, 46M05, 46M40

Editorial de la Universidad Complutense. Madrid, 1989. 
out in general. From this point of view, the notion of distinguishedness for Fréchet spaces prevents a certain "pathology", and indeed the class of the distinguished Fréchet spaces is the largest one among all classical subclasses arising in the classification of Fréchet spaces. (But note that by a very recent result of J. Taskinen [14], the Fréchet space $C(\mathbb{R}) \cap L_{1}(\mathbb{R})$, endowed with the natural intersection topology arising from the compact-open topology on $C(\mathbb{R})$ and the norm topology of $L_{1}(\mathbb{R})$, is not distinguished. Since $C(\mathbb{R}) \cap L_{1}(\mathbb{R})$ is a natural space of analysis, it is quite doubtful from this point of view whether non-distinguishedness of a Fréchet space should really be considered as a "pathology".)

The so-called "density condition" arose from the study of S. Heinrich [10] on ultrapowers of I.c. spaces. Our work in [2] on this condition started with the simple observation that for metrizable l.c. spaces, the density condition is closely related to the notion of distinguishedness. "At the end of the day", it turned out that a metrizable l.c. space $E$ satisfies the density condition if and only if the space $l_{S}(E)$ of all absolutely summable sequences in $E$ is distinguished and that this holds if and only if all bounded subsets in the strong dual $E_{b}^{\prime}$ are metrizable.

As a consequence of this remarkable equivalence, we were able to characterize the echelon spaces $\lambda_{p}=\lambda_{p}(I, A)$ (of arbitrary order $p, l \leqslant p<\infty$ or $p=0$ ) with the density condition in terms of the Köthe matrices $A$. In the case $p=1$, where the density condition is equivalent to distinguishedness, this led to a characterization of the distinguished Köthe echelon spaces $\lambda_{1}$. It was also possible to classify the distinguished Kothe echelon spaces $\lambda_{1}(E)=\lambda_{1}(I, A, E)=\lambda_{1} \hat{\otimes}_{\mathrm{r}} E$ with values in a Fréchet space $E$ completely (which involves an interesting dichotomy).

The present survey article is based on an invited lecture (by the firstnamed author) during the Functional Analysis Conference at El Escorial, June 14, 1988. In Section 1, we report on the main results of [2] and on some recent developments in this connection, mainly due to D. Vogt [16] and J. Taskinen [14]; at the end of this section, we also include a new result on the inheritance of the density condition under projective tensor products. Section 2. is devoted to a similar report on the first part of [3].

The starting point of our work in [3] was the observation that for metrizable 1.c. spaces $E$, the "proper" definitions of the notions of distinguishedness and of the density condition involve the strong dual $E_{b}^{\prime}$ of $E$ rather than $E$ itself and that several of the proofs in [2] proceeded by use of duality theory. Following the path paved by A. Grothendieck's introduction of the class of (DF)-spaces, we therefore defined and studied "dual density conditions" mainly in the framework of (DF)-spaces. (It turned out that (DF)-spaces, and not the larger class of (gDF)-spaces, are indeed the proper context for studies of this type.) 
For a strong dual of a metrizable l.c. space, quasibarrelled and bornological are equivalent notions, but these properties split up in the class of (DF)spaces. Correspondingly, we have a dual density condition $(D D C)$ and a strong dual density condition (SDDC) for (DF)-spaces. A (DF)-space satisfies (DDC) if and only if the bounded subsets of $E$ are metrizable, and it also satisfies (DDC) (resp., (SDDC)) if and only if the space $l_{\mathrm{o}}(E)$ of all bounded sequences with values in $E$ is quasibarrelled (resp., bornological).

For a locally complete (DF)-space $E$ and a Kōthe echelon space $\lambda_{1}$, (DDC) allows to characterize the (quasi-) barrelled spaces $L_{b}\left(\lambda_{1}, E\right)$ of continuous linear mappings from $\lambda_{1}$ to $E$, and to give "good" necessary and "good" sufficient conditions for $L_{b}\left(\lambda_{1}, E\right)$ to be bornological. The $\varepsilon$-tensor product $\left(\lambda_{1}\right)^{\prime} \otimes_{b} E$ and its completion $\left(\lambda_{1}\right)_{\mathrm{b}} \dot{\otimes}_{8} E$ are natural topological subspaces of $L_{\mathrm{b}}\left(\lambda_{1}, E\right)$ if $E$ is a complete l.c. space. But, interestingly enough, the dual density conditions do not occur in the classification of the quasibarrelled and bornological (DF)-spaces of type $\left(\lambda_{1}\right)_{b}^{\prime} \otimes_{E} E$ and $\left(\lambda_{1}\right)_{b}^{\prime} \dot{\otimes}_{c} E$, they come in only when one passes to the "full" space $L_{\mathrm{b}}\left(\lambda_{1}, E\right)$.

In this survey, we have concentrated on a presentation of the results of [2] and (of the first part) of [3]. Among other things, one method of [3] involves "reverse projective description", and we state the main result in this direction (as Theorem 2.7). Ideas of this type are closely connected with "weighted inductive limits" of spaces of continuous functions which, from the side of interesting applications, also were one of our main motivations for the study of the dual density conditions for (DF)-spaces. However, sketches of proofs, or of the methods, and the applications to weighted inductive limits (see the last part of [3]) are beyond the scope of this article.

\section{HEINRICH'S DENSITY CONDITION FOR FRECHET SPACES}

Ultraproducts have been used for some time in Banach space theory as a framework for some aspects of the so-called "local theory" of Banach spaces. In his article [10] (which raised a number of open questions and also introduced an interesting notion of superreflexivity for locally convex spaces), $S$. Heinrich then proceeded to study the corresponding construction in the context of general locally convex (l.c.) topological vector spaces, with applications to the nonlinear (i.e., uniform and Lipschitz) classification of such spaces.

Heinrich investigated two different kinds of ultrapowers of a l.c. space $E$ with respect to an ultrafilter $D$ on a set $I$ : the full ultrapower $(E)_{D}$ and the bounded ultrapower $[E]_{D}$, a natural subspace of $(E)_{b}$. Both definitions are canonical extensions of the Banach space concept: $(E)_{D}$ is the maximal space to which the continuous seminorms of $E$ can be extended (and arises from the nonstandard hull in nonstandard analysis), while the (new) construction of 
$[E]_{D}$ is rather based on the fact that for a Banach space $X$, the ultrapower $(X)_{D}$ is generated by all $\mathrm{D}$-bounded subsets of $X^{7} / D$. (See [10] for details.)

Both kinds of ultrapowers have certain advantages, and thus one is interested in the so-called "density condition" for a l.c. space $E$, a necessary and sufficent condition for the density of the bounded ultrapower $[E]_{D}$ in the full ultrapower $(E)_{D}$ for an arbitrary “ $\kappa(E)^{+}$-good countably incomplete" ultrafilter $D$. (The condition also occurs independently in Heinrich's investigations on the duality of ultrapowers.) Heinrich notes that "the density condition is of obvious importance in the study and application of ultrapowers" and characterizes this condition in terms of "standard" l.c. theory as follows ([10], Theorem 1.4).

1. Definition (S. Heinrich). Let $E$ denote a (Hausdorff) l.c. space, $\not L(E)$ the system of all closed absolutely convex neighborhoods of 0 in $E$ (or any basis of $O$-neighborhoods in $E)$ and $B(E)$ the system of all closed absolutely convex and bounded subsets of $E$ (or any basis of bounded sets in $E$ ).

Then $E$ satisfies the density condition if, given any function $\lambda: \mathscr{t}(E) \rightarrow \mathbb{R}_{+} \backslash\{0\}$ and an arbitrary $V \in \mathscr{W}(E)$, there always exist a finite subset $\mathbf{U}$ of $\mathscr{\ell}(E)$ and $B \in B(E)$ such that

$$
\underset{U \in U}{\cap} \lambda(U) U \subset B+V .
$$

By taking polars in the inclusion (1), it is easily seen that the I.c. space $E$ satisfies the density condition if and only if for each $\lambda: \mathscr{q}(E) \rightarrow \mathbb{R}_{+} \backslash\{0\}$ and each $V \in \mathscr{U}(E)$, one can find $\mathbf{U} \subset \mathscr{Q}(E)$ finite and $B \in B(E)$ with

$$
\Gamma\left(\cup_{U \in \mathbf{U}} \lambda(U) U^{\circ}\right)=\bar{\Gamma}\left(\cup_{U \in U} \lambda(U) U^{\circ}\right) \supset B \cap V^{\infty},
$$

where $\Gamma$ (resp., $\bar{\Gamma}$ ) denotes the absolutely convex hull (resp., the closed absolutely convex hull). The left hand side of (2) is contained in $\Gamma\left(\bigcup_{U \in t(E)} \lambda(U) U^{\circ}\right)$, a 0 -neighborhood for the inductive limit topology of $E_{i}^{\prime}=$ ind $_{t(E) \exists U} E_{U}^{\prime}$. And since the topology of $E_{i}^{\prime}$ is a priori stronger than the strong topology (of $E_{b}^{\prime}$ ), one concludes from (2) that the topologies of $E_{i}^{\prime}$ and of $E_{b}^{\prime}$ coincide on each equicontinuous subset in the dual $E^{\prime}$ of a l.c. space $E$ with the density condition.

Heinrich already noted that each quasinormable space (and thus a fortiori each (gDF)-space) as well as each Fréchet-Montel space satisfies the density condition, but that there are examples of Frechet spaces without this condition. We will therefore restrict our attention to metrizable l.c. spaces from this point on. Since the strong topology in the dual of such a space is localized to the equicontinuous (or, equivalently, strongly bounded) subsets, our previous 
discussion in connection with (2) inmediately implies the equality $E_{t}^{\prime}=E_{b}^{*}$ for each metrizable l.c. space $E$ with density condition. That is, $E_{b}{ }_{b}$ must be barrelled (or, equivalently, bornological), and hence $E$ must be distinguished (in Grothendieck's terminology). But the converse fails since there even exist reflexive Fréchet spaces without density condition.

The exact position of the class of the Fréchet spaces with density condition among other (classical) classes of Fréchet spaces can be seen from the following diagram (in which arrows stand for conclusions):

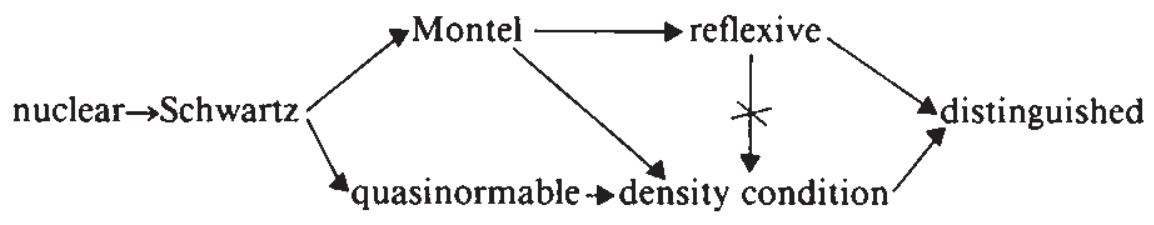

In the formulation of the subsequent (Main Characterization) Theorem 2., $l,(E)$ denotes the l.c. space of all absolutely summable sequences with values in the I.c. space $E$, and $l_{\infty}\left(E_{b}^{\prime}\right)$ is the l.c. space of all bounded sequences in $E_{b}^{\prime}$. (Also note that $l_{l}(E)$ equals the completed $\pi$-tensor product $l, \hat{\otimes}_{\Omega} \mathrm{E}$ for each Fréchet space $E$.)

2. Theorem. The following assertions for a metrizable l.c. space $E$ are equivalent:

(1) E satisfies the density condition,

(2) each bounded subset of the strong dual $E_{b}^{\prime}$ is metrizable,

(3) $l_{1}(E)$ is distinguished,

(3) $l_{\infty}\left(E_{b}^{\prime}\right)=\left(l_{1}(E)\right)_{b}^{\prime}$ is barrelled/bornological,

(3") $l_{1}(E)$ satisfies the density condition,

(3"') the bounded subsets of $l_{\infty}\left(E_{d}^{\prime}\right)$ are metrizable.

While for general Fréchet spaces $E$, the density condition is a strictly stronger assumption that the distinguishedness, it is a remarkable consequence of Theorem 2. that the two properties coincide for spaces of the form $l_{1}(E)$.

We next turn to a discussion of the density condition for echelon spaces of order $p, 1 \leqslant p<\infty$ or $p=0$, and first fix our notation: Let $A=\left(a_{n}\right)_{n}$ be a "Köthe matrix" on some (nonvoid) index set $I$; i.e., $\left(a_{n}\right)_{n}$ is an increasing sequence of strictly positive functions on $I$. For $1 \leqslant p<\infty, \lambda_{p}=\lambda_{p}(I, A)$ denotes the Fréchet space of all (real- or complex-valued) generalized sequences $x=(x(i))_{i \in 1}$ on $I$ such that

$$
\left(\sum_{i \in f}\left(a_{n}(i)|x(i)|\right)^{p}\right)^{1 / 2}<\infty \text { for each } n \in \mathbb{N} .
$$


$\lambda_{p}$ is called the echelon space of order $p$ associated with $A$, and it is the projective limit proj $l_{p}\left(a_{n}\right)$ of the "diagonal transforms" $l_{p}\left(a_{n}\right)$ of $l_{p}=l_{p}(I)$. For $p=0$, the echelon space $\lambda_{o}=\lambda_{0}(I, A)=$ proj $c_{o}\left(a_{n}\right)$ of order 0 is defined similarly, using diagonal transforms $c_{0}\left(a_{n}\right)$ of the sequence space $c_{0}=c_{0}(I)$ of all "null sequences" on $I$.

3. Theorem. An echelon space $\lambda_{p}=\lambda_{p}(I, A)$ of order $p, l \leqslant p<\infty$ or $p=0$, satisfies the density condition if and only if its Kothe matrix $A=\left(a_{n}\right)_{n}$ satisfies the following condition $(D)$ (independent of $p$ ):

There exists an increasing sequence $J=\left(I_{m}\right)_{m \in \mathbb{N}}$ of subsets $I_{m}$ of $I$ such that $(N, J)$ for each $m \in \mathbb{N}$, there is $n_{m} \in \mathbb{N}$ with inf $\frac{a_{n_{m}}(i)}{a_{i \in l_{m}}(i)}>0, k=n_{m}+1, n_{m}+2, \ldots$ while

$(M, J)$ for each $n \in \mathbb{N}$ and each $I_{\diamond} \subset I$ with $I_{o} \cap\left(\Lambda I_{m}\right) \neq \phi$ for all $m \in \mathbb{N}$, there exists $n^{\prime}=n^{\prime}\left(n, I_{d}\right)>n$ with (3) inf $\frac{a_{n}(i)}{a_{i}(i)}=0$

Condition (D) had been introduced by Bierstedt-Meise [4] (and we refer to that article for a more thorough discussion.). Roughly spoken, it requires that the index set $I$ can be written as an increasing union of subsets $I_{m}$ with the following two properties:

$(\mathrm{N}, \mathrm{J})$ The restrictions of all "weights" $a_{k}, k \geqslant n_{m}$, to $I_{m}$ induce the same weight condition.

$(\mathrm{M}, \mathrm{J})$ For any $n$ and any subset $I_{o}$ of $I$ which meets the complement of each $I_{m}$, the restrictions of $a_{n}$ and of some $a_{n^{\prime}}, n^{\prime}=n^{\prime}\left(n, I_{o}\right)>n$, to $I_{o}$ induce different weight conditions (in the sense that (3) holds).

For the classical case $p=1$ of the Kothe echelon spaces $\lambda_{1}$ (of order 1), one actually arrives at a very remarkable characterization.

4. Theorem. For a Köthe echelon space $\lambda_{1}=\lambda_{1}(I, A)$, the following assertions are equivalent:

(1) $\lambda_{1}$ is distinguished,

(2) $\lambda_{1}$ satisfies the density condition,

(3) the Kothe matrix $A=\left(a_{n}\right)_{n \in \mathbb{N}}$ of $\lambda_{1}(I, A)$ satisfies condition (D). 
(4) for every sequence $\left(D_{k}\right)_{k=\mathrm{N}}$ of positive numbers, there is a sequence $\left(C_{k}\right)_{k}$ of positive numbers such that for every $n \in \mathbb{N}$ and every $C>0$, there exists $m \in \mathbb{N}$ with

$$
\inf \left\{C a_{n^{*}} \sup \frac{a_{k}}{C_{k}} \mid \leqslant \sup \left\{\frac{a_{k}}{D_{k}} ; k \leqslant m\right\} \quad \text { (on } I\right. \text { ). }
$$

The problem of characterizing the class of the distinguished Kothe echelon spaces $\lambda_{1}=\lambda_{1}(I, A)$ in terms of the Kothe matrices $A$ was pointed out in Bierstedt-Meise-Summers [6]. In Bierstedt-Meise [4], the implication (3) $\Rightarrow(1)$ of Theorem 4. was proved. Later on, a simpler proof of $(3) \rightarrow(2)$ was given in Bierstedt-Meise [5]. Finally, by establishing the converse implications $(1) \Longrightarrow(2)$ and $(2) \Leftrightarrow(3)$, the authors derived the desired characterization $(1) \Leftrightarrow(2) \Leftrightarrow(3)$ in [2]. More recently, D. Vogt [16] noted a short direct proof of (1) $\Leftrightarrow(4)$. We also refer to $F$. Bastin [1] for a discussion of some other equivalent reformulations of (D) and of condition (4) of Theorem 4. (in the more general context of weighted inductive limits of spaces of continuous functions).

We note that an echelon space $\lambda_{p}$ of order $p, 1<p<\infty$ or $p=0$, is always distinguished and that $\lambda_{p}$ is even reflexive for $1<p<\infty$, but by Theorem 3., $\lambda_{p}=\lambda_{p}(I, A)$ satisfies the density condition if and only if $A$ satisfies condition (D). On the other hand, by Theorem 4., the Kōthe echelon space $\lambda_{1}=$ $\lambda_{1}(I, A)$ (of order 1 ) is distinguished if and only if it satisfies the density condition, and this is again governed by condition $(D)$. In particular, if the index set $I$ is countable and $\lambda_{1}=\lambda_{1}(I, A)$ is not distinguished (as it is the case for the well-known Köthe-Grothendieck example of a non-distinguished Fréchet space $\lambda_{1}$, where $I=\mathbb{N} \times \mathbb{N}$ and $A=\left(a_{n}\right)_{n}$ with

$$
\left.a_{n}(i, j)=\left\{\begin{array}{l}
j, i \leqslant n \\
1, i \geqslant n+1
\end{array}\right\}, i, j \in \mathbb{N}, n=1,2, \ldots\right),
$$

then the corresponding space $\lambda_{2}=\lambda_{2}(I, A)$ is a reflexive, separable and hilbertizable Fréchet space without density condition (so that, in its separable strong dual, one can find bounded subsets which are not metrizable).

Finally, we consider Kōthe echelon spaces $\lambda_{1}(E)=\lambda_{1}(I, A ; E)$ with values in a Fréchet space $E$. These spaces are defined in a canonical way (replacing the modulus in $\mathrm{K}=\mathbb{R}$ or $\mathrm{C}$ by the continuous seminorms of $E$ ), and we have $\lambda_{1}(E)=\lambda_{1} \hat{\otimes}_{\mathrm{z}} E$ as an aigebraic and topological equality of Fréchet spaces. The first part of the following theorem generalizes the equivalence $(1) \Leftrightarrow(3)$ of Theorem 2., while the second part provides an "individual" characterization of the distinguished spaces of type $\lambda_{1}(E)$.

5. Theorem. (a) For a Fréchet space $E$, the following assertions are equivalent: 
(1) E satisfies the density condition,

(2) for each distinguished Kothe echelon space $\lambda_{1}, \lambda_{1}(\mathrm{E})=\lambda_{1} \otimes_{n} E$ is distinguished,

(3) for each distinguished Köthe echelon space $\lambda_{1}, \lambda_{1}(E)$ satisfies the density condition.

(b) If $E$ is a Fréchet space $\neq\{0\}$ and $\lambda$, is a Kothe echelon space, then the space $\lambda_{1}(E)=\lambda_{1} \hat{\otimes}_{\mathrm{r}} E$ is distinguished, if and only if

(i) $\lambda_{1}$ is Montel and $E$ is distinguished, or

(ii) $\lambda_{1}$ is distinguished and $E$ satisfies the density condition.

Thus, while $\lambda_{1} \hat{\otimes}_{\mathrm{x}} E$ satisfies the density condition if and only if both $\lambda_{1}$ and the Fréchet space $E$ do, the characterization of the distinguished Fréchet spaces $\lambda, \hat{\theta}_{2} E$ is more delicate: Clearly, if the complete $\pi$-tensor product $\lambda_{1} \hat{\otimes}_{x} E$ is distinguished, then each of its complemented subspaces $\lambda_{1}$ and $E$ has to be distinguished, too, but this necessary condition is not sufficient, and one has to impose a stronger hypothesis on one of the spaces $\lambda_{1}$ or $E$. And while the density condition would suffice on the side of Fréchet space $E$, the Montel property (or, equivalently, reflexivity) is needed on the side of the Köthe echelon space $\lambda_{\text {; }}$ (cf. the equivalence (1) $\Leftrightarrow(2)$ in Theorem 4.).

In our article [2], we conjectured that the density condition for two general Fréchet spaces $E$ and $F$ would not even imply that $\mathrm{E} \hat{\theta}_{\mathrm{x}} \mathrm{F}$ must be distinguished, but we did not have any counterexamples at that time. Recently, as a consequence of his method of construction for counterexamples to Grothendieck's "problème des topologies", J. Taskinen [14] showed that for any Köthe echelon space $\lambda_{1}$, there is a Fréchet-Montel space $F$ such that $F \hat{\otimes}_{\mathrm{x}} F$ contains a complemented copy of $\lambda_{1}$, whence it is easy to deduce that there exist even FréchetMontel spaces $F$ such that $F \hat{\otimes}_{\mathrm{n}} F$ is not distinguished.

Then, perhaps, the right question to ask next is the following one: Is the negative solution to Grothendieck's "problème des topologies" the only obstruction to the permanence of the density condition under complete $\pi$-tensor products of Fréchet spaces? That is, must $E \hat{\otimes}_{R} F$ satisfy the density condition if both Fréchet spaces $E$ and $F$ satisfy this condition and if the pair $(E, F)$ has the property that each bounded subset $B$ of $E \hat{\theta}_{R} F$ is contained in a set of the form $\bar{\Gamma}\left(B_{1} \otimes B_{2}\right)$ with $B_{1}$ bounded in $E$ and $B_{2}$ bounded in $F$ ?

A positive answer of this question follows from the next proposition (its proof is an adaptation of the proof of Proposition 2.7 in [2]).

6. Proposition. Let E denote a metrizable l.c. space with the density condition and $F$ a l.c. space with an increasing fundamental sequence $\left(B_{n}\right)_{n \in \mathrm{N}}$ of closed absolutely convex bounded sets which are metrizable in the topology in- 
duced by $F$. Then also $L_{b}(E, F)$ has a fundamental sequence of bounded subsets which are metrizable (in the induced topology).

Proof. 1. Let $\left(U_{n}\right)_{n \in N}$ denote a decreasing basis of closed absolutely convex 0 -neighborhoods in $E$. Then it is well-known that $\left(D_{n}\right)_{n \in N}$ forms a fundamental sequence of (closed absolutely convex) bounded sets in $L_{\mathrm{b}}(E, F)$ for

$$
D_{n}:=\left\{T \in L(E, F) ; T\left(U_{n}\right) \subset B_{n}\right\}, n=1,2, \ldots
$$

(e. g., see Dierolf [8], Prop. 27.). It now suffices to show that 0 has a countable basis of neighborhoods in $D_{n}$ for the topology induced by $L_{\mathrm{b}}(E, F), n=1,2, \ldots$, and we fix $n \in \mathbb{N}$ for the rest of the proof.

2. Because of the density condition for $E, 0$ has a countable basis of neighborhoods in each $N U_{n}^{\circ} \subset E_{b}^{\prime}, N=1,2, \ldots$, and thus there exists a sequence $\left(C_{k}\right)_{k \in \mathrm{N}}$ of closed absolutely convex bounded subsets of $E$ such that for every $C \in B(E)$ and every $N \in \mathbb{N}$ there is $k \in \mathbb{N}$ with $C_{k}^{\circ} \cap N U_{n}^{\circ} \subset C^{\circ}$. In a similar way, using the fact that each $B_{n} \subset F$ is metrizable, we find a sequence $\left(V_{1}\right)_{l \in N}$ of closed absolutely convex neighborhoods of 0 in $F$ such that for every $V \in \mathscr{H}(F)$ and every $M \in \mathbb{N}$ there is $l \in \mathbb{N}$ with $V_{l} \cap M B_{n} \subset V$. At this point, it is enough to prove that for each $C \in B(E)$ and each $V \in \mathscr{Z}(F)$, there exist $k, l \in \mathbb{N}$ such that

$$
\text { (*) } \quad\left\{T \in L(E, F) ; T\left(C_{k}\right) \subset V_{i}\right\} \cap D_{n} \subset\{T \in L(E, F) ; T(C) \subset V\} \text {. }
$$

3. Given $C \in B(E)$, we first choose $M \in \mathbb{N}$ with $C \subset 2 M U_{n}$ and note that then $T(C) \subset 2 M T\left(U_{n}\right) \subset 2 M B_{n}$ for all $T \in D_{n}$. Given $V \in \forall(F)$ and $M \in \mathbb{N}$, there is $l \in \mathbb{N}$ with $V_{l} \cap M B_{n} \subset 1 / 2 V$. Next, for this $V_{t} \in \not /(F)$, we can find $N \in \mathbb{N}$ with $V_{1}^{\circ} \subset N B_{n}^{\circ}$. Finally, for the given $C \in B(E)$ and this $N \in \mathbb{N}$, there is $k \in \mathbb{N}$ with $C_{k}^{\circ} \cap 2 N U_{n}^{\circ} \subset C^{\circ}$, and taking polars in the dual system $\left\langle E_{,} E^{\prime}\right\rangle$ (and closures with respect to the topology of $E$ ), we conclude that

$$
\left.{ }^{* *}\right) C \subset C^{\circ \circ} \subset\left(C_{k}^{\circ} \cap 2 N U_{n}^{\circ}\right)^{\circ}=\bar{\Gamma}\left(C_{k} \cup \frac{1}{2 N} U_{n}\right) \overline{\subset C_{k}+\frac{1}{2 N} U_{n}} \subset C_{k}+\frac{1}{N} U_{n}
$$

To derive the desired conclusion (*), we can now proceed as follows: Fixing $T \in D_{n}$ and taking $x \in U_{n}$ and $y \in V_{i}^{\circ}$, we have $T(x) \in B_{n}$ and $y \in N B_{n}^{\circ}$, whence $|<T(x), y>| \leqslant N$, and thus $T\left(U_{n}\right) \subset N V_{i}^{\infty}=N V_{l}$ holds. If $T$ also satisfies $T\left(C_{k}\right) \subset V_{p}$ an application of $\left.{ }^{* *}\right)$ yields

$$
T(C) \subset T\left(C_{k}\right)+1 / N T\left(U_{n}\right) \subset V_{1}+\mathrm{V}_{1}=2 \mathrm{~V}_{i}
$$

But we have noted that $T(C) \subset 2 M B_{n}$ holds as well, and hence we obtain $T(C) \subset 2 V_{1} \cap 2 M B_{n} \subset V$, as desired. 
7. Corollary. Let $E$ and $F$ be Fréchet spaces with the density condition. If $E \hat{\otimes}_{\mathrm{x}} F$ has the property $(B B)$ that each bounded subset $B$ of $E \hat{\otimes}_{\mathrm{x}} F$ is contained in a set of the form $\bar{\Gamma}\left(B_{1} \otimes B_{2}\right)$ with $B_{1}$ bounded in $E$ and $B_{2}$ bounded in $F$, then $E \hat{\otimes}_{k} F$ has the density condition (and hence is distinguished).

Proof. If is well-known (e.g., see Grothendieck's thesis [9]) that, as a consequence of property $(\mathrm{BB}),\left(E \hat{\otimes}_{\mathrm{x}} F\right)_{\mathrm{b}}^{\prime}$ and $L_{\mathrm{b}}\left(E, F_{\mathrm{b}}^{\prime}\right)$ can be identified canonically. By Proposition 6. (and the equivalence (1) $\Leftrightarrow(2)$ of theorem 2.), $E \dot{\otimes}_{x} F$ must now satisfy the density condition.

From Grothendieck [9] and Taskinen [11], [13], we know that $E \hat{\otimes}_{n} F$ has property $(\mathrm{BB})$ for many pairs $(E, F)$ of Fréchet spaces. First, one can find the following cases in [9]:

(i) Both $E$ and $F$ are Banach spaces,

(ii) both $E$ and $F$ are Schwartz spaces,

(iii) $E$ or $F$ is an $\mathrm{L}^{1}$-space with respect to a measure on a locally compact space (more generally, $E$ or $F$ can be an $\mathrm{L}^{1}\left(\left(\mu_{\mathrm{i}}\right)_{i \in \mathrm{N}}\right)$-space in the sense of [9]),

(iv) $E$ or $F$ is nuclear.

Grothendieck also claims that $E \hat{\otimes}_{x} F$ satisfies (BB) if both $E$ and $F$ are hilbertisable Fréchet spaces, but he does not include a full proof of this claim, and there seems to be some doubt whether his argument is correct (cf. Taskinen [13], Section 2.4).

In [11], Taskinen proves property (BB) for projective tensor products $E \hat{\otimes}_{x} F$ of Fréchet spaces such that

(v) both $E$ and $F$ have a certain kind of basis (e.g., $p$ - or $X$-Köthe sequence spaces, where $X$ is a Banach space with a 1-unconditional basis), or $E$ is a Fréchet space with this kind of basis and $F$ is a Banach space.

He also notes that property $(\mathrm{BB})$ is inherited when one takes countable products of Fréchet spaces.

Fréchet spaces $E$ such that $E \hat{\otimes}_{x} F$ has property (BB) for each Fréchet space $F$ (resp., for each Banach space $F$ ) are called (FBB)-spaces (resp., (FBa)-spaces) in Taskinen [13], and he proceeds to study these classes. Roughly spoken, some of his results read as follows:

(vi) Fréchet-Schwartz spaces and $L\left(\left(\mu_{i}\right)_{\mathrm{i} \in N}\right)$-spaces, $1 \leqslant p<\infty$, are (FBa)spaces "provided that certain pathologies are excluded" (quote).

Moreover, $E \hat{\theta}_{\alpha} F$ definitely has property (BB) if 
(vii) $E$ is a separable hilbertisable Fréchet space and $F$ is a separable Banach space (or, more generally, $F$ is a separable ( $\mathrm{FBa}$ )-space), or if both $E$ and $F$ are separable hilbertisable Fréchet spaces.

Finally,

(viii) a Banach space is an (FBB)-space if and only if it is a $\%_{1}$-space,

and we also refer to [13], Section 3.2 for various related results.

Of course, in view of Corollary 7., each proposition on property (BB) for the projective tensor product of two Fréchet spaces $E$ and $F$ also yields a corollary on spaces $E \otimes_{\kappa} F$ wich satisfy the density condition and hence are distinguished. However, since the projective tensor product of two normed spaces is a Banach space and since Grothendieck [9] already observed that $E \otimes_{\mathrm{x}} F$ is quasinormable (resp., a Schwartz space) if (and only if) both $E$ and $F$ are quasinormable (resp., Schwartz spaces), the cases (i) and (ii) above are really 'not important in this connection (and have only been listed for purposes of completeness of the list); also note that the distinguished case of (iv) is implied by another result of Grothendieck [9] (whereby $E \hat{\theta}_{2} F$ is distinguished if one of the Fréchet spaces $E, F$ is nuclear and the other one is distinguished).

\section{THE DUAL DENSITY CONDITIONS FOR (DF)-SPACES}

In the present report on our work from [2] and [3], we have concentrated on the results which we obtained, without going into the details of the proofs. But even from our first considerations just after Definition 1.1 and from the equivalences $(1) \Leftrightarrow(2) \Leftrightarrow\left(3^{\prime}\right) \Leftrightarrow\left(3^{\prime \prime \prime}\right)$ of Theorem 1.2 , it should already be clear that duality theory played a large role in these proofs. In fact, the "right" formulation of the density condition for a metrizable l.c. space $E$ might be condition (2) of Theorem 1.2 in terms of the metrizability of the bounded subsets of the strong dual $E_{b}^{\prime}$. From this point of view, it turns out that the proper setting for many results and methods is the larger class of (DF)-spaces introduced by Grothendieck) rather than its subclass of strong duals of Fréchet spaces. In this context, however, there are two different natural candidates for a "dual density condition".

1. Definition. As before, let $E$ denote a l.c. space, $B(E)$ the system of all (closed absolutely convex) bounded subsets of $E$ and $\|(E)$ the system of all (closed absolutely convex) neighborhoords of 0 in $E$.

Then $E$ is said to satisfy the strong dual density condition (SDDC), resp. the dual density condition $(D D C)$, if, given any function $\left.\lambda: B(E) \rightarrow \mathbb{R}_{+}\right\}\{0\}$ and an arbitrary $A \in B(E)$, there always exist a finite subset $\mathrm{B}$ of $B(E)$ and $U \in \mathbb{H}(E)$ such that 


$$
A \cap U \subset \Gamma(\underset{B \in B}{\cup} \lambda(B) B), \text { resp. } A \cap U \subset \bar{\Gamma}\left(\cup_{B \in B} \lambda(B) B\right) \text {. }
$$

Note that it was irrelevant whether one took the absolutely convex hull or the closed absolutely convex hull on the left hand side of inclusion (2) in Section 1.: The sets $\lambda(U) U^{\circ}$ are absolutely convex and $\sigma\left(E^{\prime}, E\right)$-compact so that the absolutely convex hull of the finite union $\bigcup_{U \in t} \lambda(U) U^{\circ}$ is again $\sigma(E, E)$-compact and hence a fortiori closed in $E_{b}^{\prime}$ where $E$ is any l.c. space. On the other hand, in general, even a (DF)-space $E$ may not allow any fundamental sequence $B(E)$ of closed absolutely convex bounded subsets $B$ which are compact with respect to some weaker topology on $E$. Hence we do have two possibilities on the right hand side of (4) and thus the difference between (SDDC) and (DDC) in Definition 1.

It is clear from the definitions that a quasibarrelled l.c. space $E$ satisfies the density condition if and only if its strong dual $E_{s}^{\prime}$ satisfies (DDC) or, equivalenty, (SDDC), and that (SDDC) always implies (DDC). Moreover, just as every quasinormable l.c. space satifies the density condition, one easily verifies that every l.c. space with the strict Mackey convergence condition (and hence, in particular, every metrizable 1.c. space) satisfies (SDDC). And as in the class of the metrizable l.c. spaces, the density condition implies distinguishedness, each (gDF)-space with (SDDC) (resp., (DDC)) must be bornological (resp., quasibarrelled), but again the converse does not hold. Finally, due to the work of M. Valdivia [15], it is now possible to exhibit examples of (DF)-spaces with (DDC) which are not bornological and thus cannot satisfy (SDDC) so that the two classes of (DF)-spaces with (DDC) resp. (SDDC) are indeed different.

The following (Main Characterization) Theorem 2. corresponds to Theorem 1.2 and at the same time generalizes that result.

2. Theorem. Let $E$ denote a (DF)-space.

(a) The following assertions are equivalent:

(1) E satisfies the dual density condition ( $D D C$ ),

(2) each bounded subset of $E$ is metrizable,

(3) the space $l_{\infty}(\mathrm{E})$ is quasibarrelled.

(3) $l_{\infty}(E)$ satisfies (DDC).

(3') each bounded subset of $l_{\infty}(E)$ is metrizable.

(b) Similarly, the following assertions are equivalent:

(1) E satisfies the strong dual density condition (SDDC),

(2) the space $l_{\mathrm{c}}(E)$ is bornological, (2) $l_{\infty}(E)$ satisfies (SDDC). 
We recall that the 1.c. space $l_{\infty}(E)$ of all bounded E-valued sequences is a (DF)-space for every (DF)-space $E$ by a result of S. Dierolf [8]. The equivalence (a), (1) $\Leftrightarrow$ (2) justifies our terminology concerning (DDC) and (SDDC). Next, note that the assumption in Theorem 2. that $E$ is a (DF)-space cannot be weakened to the hypothesis of a (gDF)-space: For instance, the space $(C B(R), \beta)$ of all bounded continuous functions on the real line under the strict topology $\beta$ is a (gDF)-space with metrizable bounded subsets which is not quasibarrelled. Thus, the class of (DF)-spaces is indeed the proper setting for the present results, and we will restrict our attention to this class from now on.

It is possible to complete our characterization of certain l.c. properties for the (DF)-spaces of type $l_{\infty}(E)$, using a technique of A. Defant - W. Govaerts [7]

3. Corollary. If $E$ is a (DF)-space, then $l_{\infty}(E)$ is barrelled (resp., ultrabornological) if and only if $E$ is barrelled (resp., ultrabornological) and satisfies the dual density condition ( $D D C$ ) (resp., the strong dual density condition $(S D D C)$ ).

The results of Section 1 . on the echelon spaces $\lambda_{p} 1 \leqslant p<\infty$ or $p=0$; i.e., Theorems 1.3 and 1.4 , have a natural interpretation in terms of the dual coechelon spaces $K_{q}$ and $k_{q}, 1 / p+1 / q=1$ and $q=1$ for $p=0$, but this is rather trivial, and we leave it to the reader. (Our notation concerning co-echelon spaces is taken from [6].)

Thus, we immediately turn to the results in the present context which correspond to Theorem 1.5. As we have treated Köthe echelon spaces $\lambda_{1}(E)=$ $\lambda_{1}(I, A ; E)$ with values in a Fréchet space $E$ there, we can now study Köthe coechelon spaces $K_{\infty}(E)=K_{\infty}(I, \bar{V} ; F)$ and $k_{\infty}(E)=k_{\infty}(I, V ; E)$ with values in an arbitrary (DF)-space. The subsequent results are indeed valid in this general setting, and vector-valued Köthe co-echelon spaces provide a useful tool for the proofs. However, the introduction of the spaces $K_{\infty}$ and $K_{\infty}(E)$ would require several additional definitions and various comments. On the other hand, if $E$ is a locally complete l.c. space, it is easy to establish a (canonical) topological isomorphism of $K_{\infty}(E)=K_{\infty}(I, \bar{V} ; E)$ with the space $L_{b}\left(\lambda_{1}, E\right)=$ $L_{b}\left(\lambda_{1}(I, A), E\right)$ of continuous linear mappings, where $\bar{V}$ is associated with $A$. That is the reason why, for the purposes of a simpler exposition, we prefer in the sequel to formulate our results in terms of spaces of continuous linear mappings from a Köthe echelon space $\lambda_{1}$ into a locally complete (DF)-space.

We start with the following result (which can easily be deduced from the topological duality $\left(\lambda_{\mathrm{r}}(F)\right)_{b}^{\prime}=\left(\lambda_{1} \hat{\otimes}_{n} F\right)_{b}^{\prime}=K_{\infty}(E)$ if the space $E$ is the strong dual $F_{b}^{\prime}$ of some Fréchet space $F$, and) which should be compared with the examples (due to J. Taskinen [12]) of Fréchet-Montel spaces $F$ and Banach spaces $E$ such that $L_{b}(F, E)$ fails to be a (DF)-space. 
4. Proposition. If $E$ is a locally complete l.c. space, then $L_{b}\left(\lambda_{1}, E\right)$ is a $(D F)$ space if and only if $E$ is.

Next, we come to the analog of Theorem 1.5. Part (a) of this theorem generaiizes Theorem 2. above (in view of the topological isomorphism $l_{\infty}(E)=$ $L_{b}\left(l_{1}, E\right)$ for locally complete 1.c. spaces $\left.E\right)$. Also note that the characterization of the locally complete (quasi-) barrelled (DF)-spaces of type $L_{b}\left(\lambda_{1}, E\right)$ in Theorem 5.(b) exhibits the same dichotomy which we already know from Theorem 1.5.(b).

5. Theorem. (a) For a locally complete (DF)-space E, the following assertions are equivalent:

(1) E satisfies $(D D C)$ (resp., (SDDC)),

(2) for each distinguished Köthe echelon space $\lambda_{1}$, the space $L_{b}\left(\lambda_{1}, E\right)$ is quasibarrelled or, equivalently, barrelled (resp., bornological or, equivalently, ultrabornological),

(3) for each distinguished Köthe echelon space $\lambda_{1}$, the space $L_{b}\left(\lambda_{1}, E\right)$ satisfies $(D D C)$ (resp., $(S D D C)$ ).

(b) If $E$ is a locally complete (DF)-space $\neq\{0\}$ and $\lambda_{1}$ is a Kothe echelon space, then $L_{b}\left(\lambda_{1}, E\right)$ is quasibarrelled (or, equivalently, barrelled) if and only if

(i) $\lambda_{1}$ is Montel and $E$ is (quasi-) barrelled, or

(ii) $\lambda_{1}$ is distinguished and $E$ satisfies (DDC).

One can conjecture that the result corresponding to Theorem 5.(b) with $L_{b}\left(\lambda_{1}, E\right)$ and $E$ bornological (instead of barrelled) and (SDDC) (instead of (DDC)) for $E$ is also valid. Unfortunately, we are not able to prove such an individual characterization of the locally complete bornological (DF)-spaces of type $L_{b}\left(\lambda_{1}, E\right)$ at the moment. But we do have partial results with a (small) gap between the necessary and the sufficient conditions.

6. Proposition. Let $E \neq\{0\}$ denote a locally complete $(D F)$-space. If $L_{b}\left(\lambda_{1}, E\right)$ is bornological, then $\lambda_{1}$ has to be distinguished and $E$ has to be bornological; moreover, if $\lambda_{1}$ is not Montel, then E must definitely also satisfy (SDDC). Conversely, if $\lambda_{1}$ is distinguished and $E$ satisfies (SDDC), but also if $\lambda_{1}$ is a Schwartz space and $E$ is a bornological space such that each sequence converg. ing to zero is a local null sequence, then $L_{b}\left(\lambda_{1}, E\right)$ is again bornological.

We do not intend to give a sketch of proof of Theorem 5. or Proposition 6. here, but we would like to outline one approach to results of this type since it also serves to put them in the proper perspective. Let us recall that $\lambda_{1}=$ $\lambda_{3}(I, A), A=\left(a_{n}\right)_{n}$, is nothing but the projective limit $\underset{-n}{\operatorname{proj}} l_{f}\left(a_{n}\right)$ of the diagonal 
transforms $l_{1}\left(a_{n}\right)$ of the space $l_{1}=l_{1}(I)$. From this point of view the following theorem answers a natural question wich comes up in this context.

7. Theorem. Let $E$ denote a locally complete l.c. space with the countable neighborhood property (e.g., E can be a locally complete ( $g D F)$-space) and $\lambda_{1}$ a distinguished Kothe echelon space. Then we have a (canonical) topological isomorphism

$$
L_{b}\left(\lambda_{1}, E\right)=L_{b}\left(\operatorname{proj}_{-n} l_{1}\left(a_{n}\right), E\right)=\operatorname{ind}_{n \rightarrow} L_{b}\left(l_{1}\left(a_{n}\right), E\right)\left(=\operatorname{ind} l_{\infty}\left(v_{n}, E\right)=k_{\infty}(I, V ; E),\right.
$$

where $l_{\infty}\left(v_{n} E\right)$ denotes the diagonal transform of the space $l_{\infty}(E)=l_{\infty}(I ; E)$ of all bounded E-valued generalized sequences on I by the function

$$
\left.v_{n}=1 / a_{n}, n \in \mathbb{N}\right) \text {. }
$$

In the context of the vector-valued Köthe co-echelon spaces $K_{\mathrm{o}}(E)=$ $K_{\infty}(I, \bar{V} ; E)$ and $k_{\infty}(E)=k_{\infty}(I, V ; E)$, Theorem 7. is a "projective description result" for $k_{\infty}(E)$ (and it also holds more generally for weighted inductive limits of spaces of continuous E-valued functions, of. Section 4. of [3]).

It is now quite interesting to compare Theorem 5. and Proposition 6. for $L_{b}\left(\lambda_{1}, E\right), E$ locally complete (DF), with similar results for the topological linear subspace $\left(\lambda_{1}\right)_{b}{ }_{b} \otimes_{e} E$ of all mappings in $L_{b}\left(\lambda_{1}, E\right)$ with finite dimensional range, respectively for the completion $\left(\lambda_{1}\right)_{b}^{\prime} \dot{\theta}_{2} E$ of this tensor product, which coincides with the subspace of all those mappings in $L_{b}\left(\lambda_{1}, E\right)$ for which the image of each bounded set in $\lambda_{1}$ is precompact in $E$ whenever $E$ is complete. In fact, it turns out that the dual density conditions do not occur in the context of the $\varepsilon$-tensor products, and that they come in only when one passes from the corresponding subspaces to all of $L_{b}\left(\lambda_{1}, E\right)$. This phenomenon also serves to explain one aspect of the dichotomy in Theorem 5 . (b) in a very satisfactory way. We collect the main results in our final theorem.

8. Theorem. (a) If $\lambda_{1}$ is an arbitrary Köthe echelon space and $E$ is $a(D F)$ space, the $\varepsilon$-tensor product $\left(\lambda_{1}\right)_{b}^{\prime} \otimes_{\mathrm{E}} E$, and hence also its completion $\left(\lambda_{1}\right)_{b}^{\prime} \dot{\otimes}_{\varepsilon} E$, is again a $(D F)$-space.

(b) For a Kothe echelon space $\lambda_{1}$ and $a(D F)$-space $E,\left(\lambda_{1}\right)_{b} \otimes_{a} E$ is quasibarrelled (resp., bornological) if and only $\lambda_{1}$ is distinguished and $E$ is quasibarrelled (resp., bornological).

(c) If $\lambda_{1}$ denotes a Kothe echelon space and $E$ a complete (DF)-space, the following conditions are necessary and sufficient for the barrelledness of $\left(\lambda_{1}\right)_{\mathrm{b}} \dot{\otimes}_{\mathrm{B}} E: \lambda_{1}$ is distinguished and $E$ is barrelled.

The case of the complete bornological $(D F)$-spaces of type $\left(\lambda_{t}\right)_{b}{ }_{b} E$ again presents some technical difficulties, and we only state the following partial results: 
Let $\lambda_{1}$ be a Köthe echelon space and $E$ a complete (DF)-space. $\left(\lambda_{1}\right)_{b}^{\prime} \dot{\theta}_{E} E$ bornological clearly implies $\lambda_{1}$ distinguished and $E$ bornological. Conversely, if $\lambda_{1}$ is quasinormable and $E$ is a bornological space such that every absolutely convex compact subset $K$ of $E$ is "very compact", in the sense that there is an absolutely convex bounded set $B \subset E$ such that $\mathrm{K}$ is (contained and) compact in $E_{B},\left(\lambda_{\mathrm{b}}\right)_{b}^{\prime} \bar{\otimes}_{\varepsilon} E$ is (ultra-) bornological.

In concluding, we remark that Proposition 6. of Section 1. has the following obvious consequence:

9. Proposition. Let $E$ denote a metrizable l.c. space with the density condition and $F a(D F)$-space with $(D D C)$. If $L_{b}(E, F)$ is again a (DF)-space, then $L_{b}(E, F)$ satisfies (DDC) (and hence is quasibarrelled).

In some sense, this proposition generalizes Corollary 1.7. (Note that property (BB) for the projective tensor product $E \hat{Q}_{n} F$ of two Fréchet spaces implies that $L_{b}\left(E, F_{b}^{\prime}\right)$ is a (DF)-space, and even the strong dual of a Fréchet space.)

Finally, we remark that the general hypotheses of this section; viz., that $E$ can be an arbitrary (DF)-space (but it is not required that $E$ is the strong dual of some Fréchet space), allow to apply the results e.g. to countable inductive limits of normed spaces. In particular, if an (LB)-space $E=$ ind $E_{n}$ is boundedly retractive; i.e., if for each bounded subset $B$ of $E$, there is an index $n=n(B) \in \mathbb{N}$ such that $B$ is contained and bounded in $E_{n}$ and such that the topologies of $E$ and $E_{n}$ coincide on $B$, then $E$ clearly satisfies (DDC) since all bounded subsets are metrizable. but one can easily show by using the equivalence $(1) \Leftrightarrow(2)$ of Theorem 2.(b) that it even satisfies (SDDC). (If $E=$ ind $E_{n}$ is a countable injective boundedly retractive l.c. inductive limit of (DF)-spaces, then $l_{\infty}(E)=$ ind $l_{\infty}\left(E_{n}\right)$ holds algebraically and topologically.)

In fact, originally, our main motivation for the introduction of the dual density condition in the context of (DF)-spaces and for the work on which we have reported in this section were interesting applications to weighted inductive limits $V C(X)$ of spaces of the continuous functions. Howewer, an account of these applications is beyond the scope of the present (introductory) survey, and we have to refer to the last part of our original article [3].

\section{References}

[1] F. Bastin, On bornological spaces $\mathrm{CV}(\mathrm{X})$, Arch. Math. 53 (1989), 394-398.

[2] K. D. BIERSTEDT, J. BONET, Stefan Heinrich's density condition for Fréchet spaces and the characterization of the distingtished Köthe echelon 
spaces, Math. Nachr. 135 (1988), 149-180. Also see: La condition de densité et les espaces échelonnés distingués, C.R. Acad. Sci. Paris, t. 303, Série $\mathrm{I}, \mathrm{n}^{\circ} 10,1986$, pp. 459-462.

[3] K. D. BIERSTEDT, J. BONET, Dual density conditions in (DF)-spaces, I, Results Math. 14 (1988), 242-274; II, Bull. Soc. Roy. Sci. Liège 57 (1988), 567-589.

[4] K. D. BIERSTEDT, R. MEISE, Distinguished echelon spaces and the projective description of weighted inductive limits of type $V_{d} C(X)$, pp. 169-226 in: Aspects of Mathematics and its Applications, Elsevier Science Publ. B.V., North-Holland Math. Library, 1986.

[5] K. D. BIERSTEDT, R. MEISE, Weighted inductive limits and their projective descriptions, Doğa Tr. J. Math. 10, 1 (1986) (Special Issue: Proceedings of the Silivri Conference 1985), 54-82.

[6] K. D. BIERSTEDT, R. G. MEISE, W. H. SUMMERS, Kothe sets and Kothe sequence spaces, pp. 27-91 in: Functional Analysis, Holomorphy and Approximation Theory, North-Holland Math. Studies 7I, 1982.

[7] A. DEFANT, W. GOVAERTS, Tensor products and spaces of vector-valued continuous functions, Manuscripta Math. 55 (1986), 433-449.

[8] S. DIEROLF, On spaces of continuous linear mappings between locally convex spaces (Habilitationsschrift, Univ. München 1983), Note di Matematica 5 (1985), 147-255.

[9] A. GROTHENDIECK, Produits tensoriels topologiques et espaces nucléaires, Mem. Amer. Math. Soc. 16 (1955, reprint 1966).

[10] S. HEINRICH, Ultrapowers of locally convex spaces and applications I, Math. Nachr. 118 (1984), 285-315; II, Math. Nachr. 121 (1985), 211-229.

[11] J. TASKINEN, Counterexamples to "problème des topologies" of Grothendieck, Ann. Acad. Sci. Fenn., Serie A, no. 63, 1986.

[12]. J. TASKINEN, The projective tensor product of Fréchet Montel spaces, Studia Math. 91 (1988), 17-30.

[13] J. TASKINEN, $(F B a)$-and (FBB)-spaces, Math. Z. 198 (1988), 339-365.

[14] J. TASKINEN, Examples of non-distinguished Fréchet spaces, to appear in Ann. Acad. Sci. Fenn.

[15] M. VALDIVIA, $A$ class of quasibarrelled (DF)-spaces which are not bornological, Math. Ann. 136 (1974), 249-251.

[16] D. VOGT, Distinguished Kothe spaces, Math. Z. 202 (1989), 143-146.

FB 17, Mathematik,

Universităt-GH-Paderborn,

Postfach 1621 ,

D-4790 Paderborn,

Federal Republic of Germany.
Departamento de Matemáticas,

E.T.S. Arquitectura,

Universidad Politécnica de Valencia,

Camino de Vera,

E-46071 Valencia, Spain 